sensitive and accurate disease activity measures. This is a top barrier, both for treat-to-target management of SLE patients in clinical practice, as well as in clinical trials for new SLE treatments. ${ }^{12}$

The Physician's Global Assessment (PGA) is a pragmatic disease activity measure, using a $100 \mathrm{~mm}$ visual analogue scale (VAS) for physicians to quantify the patient's overall disease activity in a $0-3$ range. The PGA is sensitive to change, but it requires an experienced physician to use it reliably and consistently in the follow-up of individual patients; furthermore, it has a high interobserver variability. Despite these pitfalls, the PGA is widely applied, including in the composite responder indexes used as primary outcome measure in SLE clinical trials (i.e. SLE Responder Index [SRI] and BILAG-Based Composite Lupus Assessment [BICLA]), the proposed definitions for clinical remission (e.g. DORIS), and low disease activity states (i.e. LLDAS). ${ }^{1}$

The SLE Disease Activity Index (SLEDAI), with 24 items, is an extensively used disease activity measure, both in clinical practice and in clinical trials, where it is the driver of clinical response in the SRI. However, SLEDAI presents low sensitivity to change in disease activity - $14.9-47.4 \%$ for improvement and $26.2-59.1 \%$ for worsening. ${ }^{3} 4$ As a result, usefulness of SLEDAI for guiding treatment decisions in clinical practice is limited. Furthermore, its use for defining responders with the SRI leads to low efficiency of clinical trials. ${ }^{1}$

The BILAG is an organ-based instrument that classifies disease activity separately in nine organ-systems and includes 97 items. Due to its high complexity it is not widely used in clinical practice. It is a component of SRI and BICLA. However, it consolidates all features within an organ-system into a single score and thresholds defined for category change impede interpretation. In SRI it is applied only to guarantee specificity of clinical response that is defined by a 4-point decrease in the SLEDAI. The usefulness of BILAG in SRI is unclear, as SLEDAI shows a specificity for change over $90 \%$ and in clinical trials few patients improving in SLEDAI presented deterioration in BILAG. ${ }^{1} 34$

The SLE Disease Activity Score (SLE-DAS) is a recently validated 17-item composite index with continuous measurement properties. As compared to SLEDAI-2K, the SLE-DAS presented higher accuracy in measuring SLE disease activity and better sensitivity-to-change, as well as higher predictive value for damage accrual. ${ }^{5}$ Advantages of SLE-DAS include its continuous nature and the inclusion of important disease activity features absent from SLEDAI. Ongoing research suggests that SLE-DAS can accurately define clinical remission, low disease and active disease categories, with a more practical definition compared to other instruments.

\section{Learning Objectives}

- Identify unmet needs for measuring SLE disease activity in clinical practice and clinical trials

- Understand the strengths and pitfalls of instruments to measure SLE disease activity

- Apply at each clinical context the most appropriate instrument for measuring disease activity

\section{REFERENCES}

1. Dall'Era M, Bruce IN, Gordon C, et al. Current challenges in the development of new treatments for lupus. Ann Rheum Dis 2019;78(6):729-35.

2. Manzi S, Raymond S, Tse K, et al. Global consensus building and prioritisation of fundamental lupus challenges: the ALPHA project. Lupus Sci Med 2019;6(1): e000342.
3. Jesus $D$, Rodrigues $M$, Matos $A$, et al. Performance of SLEDAl-2K to detect a clinically meaningful change in SLE disease activity: a 36-month prospective cohort study of 334 patients. Lupus 2019;28(5):607-12.

4. Yee CS, Farewell VT, Isenberg DA, et al. The use of Systemic Lupus Erythematosus Disease Activity Index-2000 to define active disease and minimal clinically meaningful change based on data from a large cohort of systemic lupus erythematosus patients. Rheumatology (Oxford) 2011;50(5):982-8.

5. Jesus D, Matos A, Henriques C, et al. Derivation and validation of the SLE Disease Activity Score (SLE-DAS): a new SLE continuous measure with high sensitivity for changes in disease activity. Ann Rheum Dis 2019;78(3):365-71.

\section{LIFESTYLE FOR LUPUS PATIENTS: EXERCISE, DIET, AND WELL-BEING: PERSPECTIVES FROM CLINICAL PRACTICE}

Chiara Tani. University of Pisa, Italy

\subsection{6/lupus-2020-la.5}

As systemic lupus erythematosus (SLE) is a chronic condition with a significant impact on physical and mental health, all potential interventions to improve quality of life are relevant for SLE patients.

Among the possible non-pharmacological interventions, exercise and diet have a pivotal role. ${ }^{1}{ }_{2}^{2}$ For SLE patients aerobic exercise programs are safe and effective on improving aerobic and functional capacity, in addition to tolerance to exercise. Physical activity enhances cardiovascular well-being in SLE patients by reducing their body weight and waist circumference, while improving their maximum oxygen consumption, endothelial function and insulin sensitivity. Moreover, several studies also suggest a reduction in fatigue and a beneficial effect of exercise on depression, anxiety, pain, poor-quality sleep and, more generally, on health-related quality of life (HRQoL).

Diet can have a role in reducing modifiable cardiovascular risk factors; a benefit on SLE activity and HRQoL is also reported. The most robust evidence is available for polyunsaturated fatty acids enriched diets; however, we cannot draw definitive conclusions on whether there is any kind of diet that is better than another for SLE patients. Patients with SLE who smoke have an increased risk of disease flare, particularly skin manifestations, a poorer HRQoL and a reduced response to antimalarial drugs. The more intense and the longer tobacco consumption is the higher the risk of poor control of disease activity. Therefore, these facts can provide additional motivation for patients to stop smoking.

Alongside clinical advantages, evidence-based activities promoting healthy lifestyles could contribute both to patient empowerment in disease self-management and to the sustainability of public health services.

\section{Learning Objectives}

- Describe the link between lifestyles, disease activity and longterm outcomes in SLE

- Discuss the current state of studies on non-pharmacological interventions in SLE

\section{REFERENCES}

1. Fangtham $M$, Kasturi $S$, Bannuru RR, et al. Non-pharmacologic therapies for systemic lupus erythematosus. Lupus 2019;28(6):703-12.

2. Rodríguez Huerta MD, Trujillo-Martín MM, Rúa-Figueroa l, et al. Healthy lifestyle habits for patients with systemic lupus erythematosus: A systemic review. Semin Arthritis Rheum 2016;45(4):463-70. 